\title{
The Extent of Adaptation in Bullfrog Saccular Hair Cells
}

\author{
Gordon M. G. Shepherd' ${ }^{1}$ and David P. Corey ${ }^{1,2,3}$ \\ 'Program in Neuroscience, Harvard Medical School, ${ }^{2}$ Neuroscience Group, Howard Hughes Medical Institute, and \\ ${ }^{3}$ Department of Neurology, Massachusetts General Hospital, Boston, Massachusetts 02114
}

\begin{abstract}
Positive deflection of the sensory hair bundle of a vertebrate hair cell opens transduction channels to depolarize the cell. In bullfrog saccular hair cells, there is a subsequent adaptation process, whereby the proportion of transduction channels that are open, and thus the receptor current, declines toward the resting value. This occurs because the sensitivity curve, relating open probability to bundle deflection, shifts along the deflection axis in response to bundle deflections, in a manner consistent with a relaxation of mechanical tension on transduction channels. In this study we determined the extent of adaptation, measured as the shift of the sensitivity curve following deflection of the hair bundle. The shift was determined both by comparison of the receptor current in the adapted state to the resting sensitivity curve, and by comparison of pre- and postadapted sensitivity curves. The adaptive shift approached steady state with a time constant of 20-30 msec, and was at steady state within $150 \mathrm{msec}$. For all positive and for small negative deflections, both methods showed a shift that was $\sim 80 \%$ of the deflection. For larger negative deflections, the shift reached a fixed limit that was $100-500 \mathrm{~nm}$ negative to the freestanding bundle position. The limited extent of adaptation confers a timedependent sensitivity: the cell has an instantaneous or phasic sensitivity curve that is steep, and steady-state or tonic sensitivity curve that is about five times broader. It also suggests the existence of two additional structural elements within the transduction apparatus. A revised quantitative theory accommodates these elements.
\end{abstract}

[Key words: sensory transduction, sensory adaptation, mechanical sensitivity, hair cell auditory system, vestibular system, sacculus]

The mechanical transduction system in vertebrate hair cells responds, on a time scale of tens of microseconds, to extremely small deflections of the stereociliary bundle (for reviews, see Hudspeth, 1989; Pickles and Corey, 1992). The sensitive range of hair cells, taken as the nonsaturated part of the currentdeflection or " $I(X)$ " curve, generally measures $50-500 \mathrm{~nm}$ in species such as frog and turtle, and behavioral thresholds are more than an order of magnitude smaller (see Hudspeth, 1989). The conversion of mechanical to electrical signals relies on the

\footnotetext{
Received July 19, 1993; revised Mar. 21, 1994; accepted Apr. 21, 1994.

We thank Drs. J. A. Assad, S. C. Cannon, R. A. Eatock, M. C. Liberman, E. Raviola, B. J. Schnapp, and C. K. Solc for their comments on the manuscript. This work was supported by the Howard Hughes Medical Institute, NIH Grant DC00304, and Office of Naval Research Grant N00014-91-J-1159.

Correspondence should be addressed to David P. Corey, Wellman 414, Massachusetts General Hospital, Fruit Street, Boston, MA 02114.
}

Copyright (C) 1994 Society for Neuroscience $0270-6474 / 94 / 146217-13 \$ 05.00 / 0$ activation of transduction channels by tension in "gating springs" (Corey and Hudspeth, 1983b; Howard and Hudspeth, 1988), which probably correspond to the tip links at the distal ends of stereocilia (Pickles et al., 1984; Assad et al., 1991).

The transduction mechanism also adapts, on a time scale of tens of milliseconds, to sustained deflections of the bundle. Adaptation acts by shifting the $I(X)$ curve in the direction of the stimulus, thus compensating for static and potentially saturating deflections (Corey and Hudspeth, 1983a; Eatock et al., 1987; Assad et al., 1989; Crawford et al., 1989; Hacohen et al., 1989; Assad and Corcy, 1992). The adaptation mechanism also provides resting tension to the channels, setting their steady-state open probability at a steep region of the $I(X)$ curve and allowing them to register both positive and negative deflections. In bullfrog saccular hair cells, adaptation is essentially mechanical, possibly involving an adjustment in the position of one end of the tip link to restore it to a constant tension (Howard and Hudspeth, 1987; Assad and Corey, 1992). The accumulated evidence suggests that this is an active process, most likely mediated by a mechanoenzyme (Assad et al., 1989; Assad and Corey, 1992; Gillespie and Hudspeth, 1993).

The existing adaptation model (Howard and Hudspeth, 1987; Assad and Corey, 1992) supposes that steady-state gating-spring tension is set by a balance between slipping and climbing. Unless the balance is perturbed, for example, by changing the calcium ion concentration, steady-state tension is independent of bundle position in this model. There is no intrinsic set point, and adaptation is complete; the shift of the $I(X)$ curve equals the bundle deflection. There is reason to suspect that there is a set point, however. Experimentally, it is common practice to make delicate adjustments in the probe position, to obtain the most sensitive responses. Also, it is evident in most preparations that adaptation fails to return the receptor current completely to the resting level. Incomplete adaptation is noticeable in hair cells from bullfrog sacculus (Corey and Hudspeth, 1983a; Eatock et al., 1987; Howard and Hudspeth, 1988; Hacohen et al., 1989; Assad and Corey, 1992), bullfrog utriculus (Baird, 1992), turtle basilar papilla (Crawford et al., 1989), mouse cochlea (Kros et al., 1992), and chick auditory organ (Kimitsuka and Ohmori, 1992).

We measured the extent of adaptation, defined as the shift of the $I(X)$ curve following bundle deflection, to determine its time course and steady-state value. In bullfrog saccular hair cells bathed in $4 \mathrm{mM} \mathrm{Ca}^{2+}$, adaptation reaches its final extent within 150 msec. It occurs with a constant efficiency, as a proportion of the bundle deflection, of $\sim 80 \%$ over most of its range, but there is also a negative saturation that limits negative-going adaptation to less than about $-500 \mathrm{~nm}$. Physiologically, the incompleteness of adaptation would endow the hair cell with a 


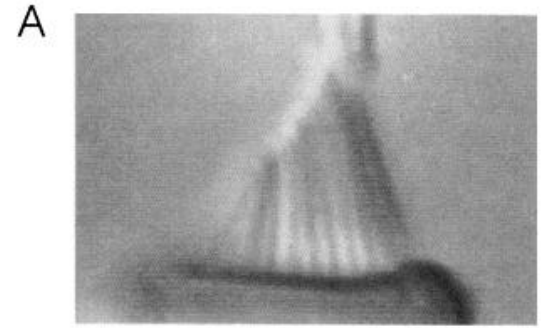

B

Figure 1. Resiliency of transduction and adaptation. Bundles were deflected from the resting position $(A)$ to extreme positive $(B)$ or negative $(C)$ positions. The sensitivity, measured at the resting position with a fast triangle-wave stimulus, was the same before (light current trace in $D$ ) and after (bold current trace in $D$ ) the procedure. Adaptation also survived, as shown by the family of responses in $E$.

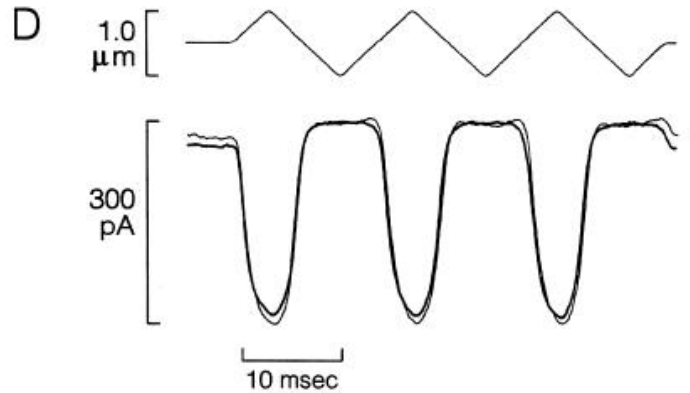

E

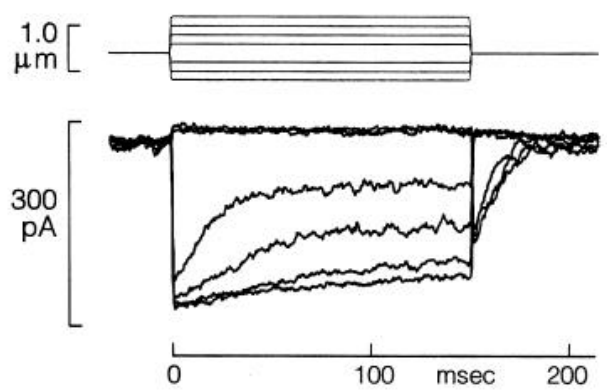

low steady-state sensitivity to mechanical stimuli, in addition to the high instantaneous sensitivity characterized previously.

Some of these results have appeared in preliminary form (Shepherd et al., 1991).

\section{Materials and Methods}

Electrophysiological recording and mechanical stimulation. As previously described (Assad and Corey, 1992), single hair cells were dissociated from the sacculi of adult bullfrogs (Rana catesbeiana), and membrane currents were measured with standard whole-cell patch-clamp methods, at a holding potential of $-80 \mathrm{mV}$. The pipette solution contained (in mM) $120 \mathrm{CsCl}, 2 \mathrm{MgCl}_{2}, 10$ EGTA, $2 \mathrm{Na}_{2} \mathrm{ATP}$, and $5 \mathrm{HEPES}$. The bath solution contained (in mM) $120 \mathrm{NaCl}, 2 \mathrm{KCl}, 4 \mathrm{CaCl}_{2}, 5 \mathrm{CsCl}$, 3 dextrose, and 5 HEPES, oxygenated at $22^{\circ} \mathrm{C}$. Stimuli were generated and responses recorded and analyzed with an 80486-based computer and laboratory interface (LM-900, Dagan) running the programming language AxoBasic (Axon Instruments). Receptor currents were sampled at $2 \mathrm{kHz}$. Individual hair bundles were deflected by a one-dimensional piezoelectric bimorph stimulator whose $10-90 \%$ rise time was below $1.0 \mathrm{msec}$ (Corey and Hudspeth, 1980); a two-dimensional stimulator that settled in under $2.0 \mathrm{msec}$ was used in a few early experiments. Hollow glass stimulus probes were coupled to the kinociliary bulbs of hair cells with light suction (Holton and Hudspeth, 1986). Care was taken to engage bundles at their freestanding position. To present stimulus protocols away from the initial resting position, the bundle was biased by manual adjustment of the micromanipulator (Physik Instrumente, Germany). Cells were imaged at $10,000 \times$ with a CCD video camera, and all stimulus presentations were recorded onto videotape.

Stimulus protocols. Adaptation at short times was studied with a family of eight $150 \mathrm{msec}$ adapting steps, to $0,200,-200,400,-400$, $600,-600$, and $800 \mathrm{~nm}$. The interval between steps was $650 \mathrm{msec}$, and the entire protocol lasted $6.4 \mathrm{sec}$. Adaptation at longer times was studied with a family of twenty $4.0 \mathrm{sec}$ adapting steps, whose amplitudes grew from $\pm 100 \mathrm{~nm}$ to $\pm 1000 \mathrm{~nm}$ in $100 \mathrm{~nm}$ increments, alternating between positive and negative values. The interval between adapting steps was a $4.0 \mathrm{sec}$ baseline step, and the entire protocol lasted $160 \mathrm{sec}$. During the last $1.5 \mathrm{sec}$ of each adapting and baseline step, a set of 16 test deflections was presented to measure the instantaneous $I(X)$ relation. These test deflections lasted $10 \mathrm{msec}$, and were spaced at $90 \mathrm{msec}$ to allow recovery from any adaptation they caused. For baseline $I(X)$ curves, the test deflection amplitudes were $100,0,200,-100,300$, $-200,400,-300,500,-400,600,-500,900,-800,1200$, and -1100 $\mathrm{nm}$. To ensure adequate sampling for adapted $I(X)$ curves, the test step pattern was broadened and shifted by half the value of the adapting step magnitude.

Analysis of current traces. Experiments were analyzed only if the videotape showed firm coupling of the probe and bundle, and very little or no rocking of the bundle or cell, since either would mimic adaptation and increase its apparent extent. The tight adhesion of the cells to the clean glass coverslip, plus the stabilization provided by positioning the patch electrode near the cuticular plate, usually prevented rocking. Extremely large test deflections sometimes induced a tiny amount of rocking, but from video records we estimated that it was $<5 \%$ of the bundle deflection at worst, and much less or undetectable for the majority of cells. Traces were analyzed only for cells with $\geq 100 \mathrm{pA}$ of transduction current at $-80 \mathrm{mV}$ (in $4 \mathrm{mM} \mathrm{Ca}^{2+}$ ). Most currents were $150-300 \mathrm{pA}$, and the largest were $\sim 400 \mathrm{pA}$. A few traces were filtered at $1-2 \mathrm{kHz}$ by digital gaussian filtering before further analysis.

Current values for $I(X)$ plots were obtained by averaging over selected regions of traces. For the $150 \mathrm{msec}$ adapting steps, instantaneous current values were obtained by sampling over the $1-2$ msec encompassing the peak current levels, and steady-state current values were obtained by averaging the last $10-20 \mathrm{msec}$ of the step response. These short-duration current traces were also used for the inferred-shift method, as described below. For the $4.0 \mathrm{sec}$ adapting steps, the region for averaging was selected individually. Steady-state current levels were obtained by averaging over a selected $\sim 0.4 \mathrm{sec}$ region starting 1.0-1.4 sec after step deflection.

Curve-fitting procedures. Instantaneous $I(X)$ relations were fitted with a form of the Boltzmann distribution for a three-state system (Corey and Hudspeth, 1983b):

$$
\begin{aligned}
p_{o}= & \frac{1}{1+\exp \left[N \Delta U_{12}^{0}-Z_{12}\left(X_{s}-X_{e}\right)\right] / k_{\mathrm{B}} T \times} \\
& \left\{1+\exp \left[N \Delta U_{01}^{0}-Z_{01}\left(X_{s}-X_{e}\right)\right] / k_{\mathrm{B}} T\right\}
\end{aligned}
$$

Channel open probability is $p_{o}, N$ is the number of channels per cell, $X_{s}$ is bundle position, $X_{e}$ is the extent of adaptation, and $k_{\mathrm{B}} T$ is the Boltzmann constant times temperature (taken at room temperature). The $\Delta U_{i j}^{0}$ terms represent the intrinsic (zero-deflection) energy difference of each channel between the states $i$ and $j$, and the $Z_{i j}$ terms represent the deflection sensitivity of the energy difference. Curves were fitted by least-squares minimization. A manual fitting procedure proved most expedient, particularly when augmented with an audio tone whose calculated frequency represented the sum of the squared differences. Average values of the parameters for a sample of 14 cells in these exper- 
A

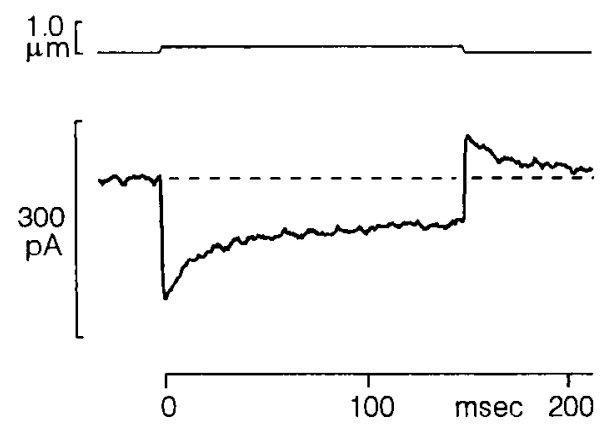

B

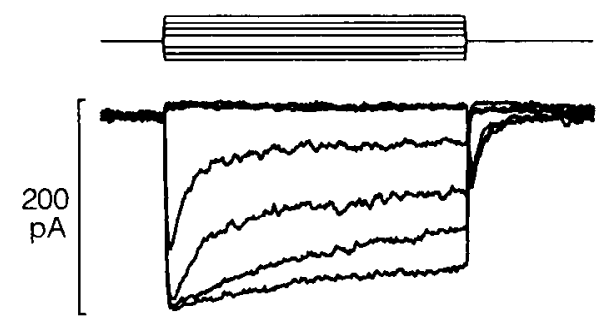

C
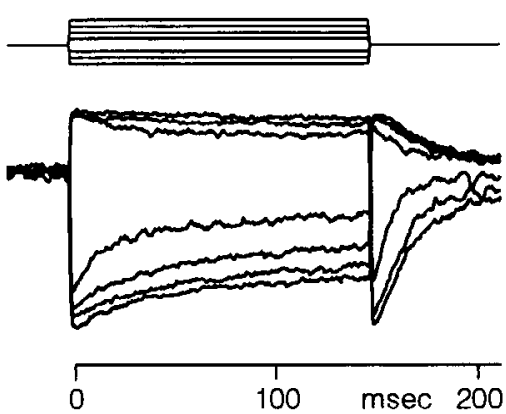

D

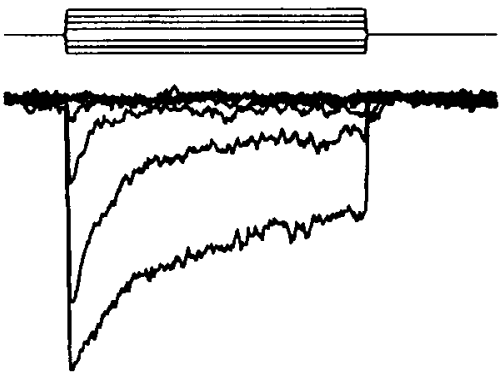

Figure 2. Qualitative features of adaptation. $A$, Typical response to a step deflection ( $+200 \mathrm{~nm}, 150 \mathrm{msec}$ ), showing the characteristic return of the receptor current toward the resting level (dashed line) after the instantaneous responses to both the onset and offset of the stimulus. $B$, Typical family of responses to step deflections varying in amplitude. Larger deflections elicited larger initial currents up to a saturating step size, and the ensuing adaptation process appeared to be increasingly incomplete. Rebound transients generated at the offset of negative steps reflect the extent of adaptation during the step; here, they mostly overlapped and, at their peak, reached only a fraction of the maximal current. $C$, A cell with a different pattern of responses to the same stimulus family, in which the resting current was a larger proportion of the maximum, and the rebounds following negative deflections indicated greater negative-going adaptation (same cell as in $A$ ). $D$, This cell had almost no resting current, and almost no rebound transients. Traces are averages of 3-12 traces. Deflection scale in $A$ applies to all panels; current scales in $A$ and $B$ apply to $C$ and $D$, respectively. iments were $N \Delta U_{01}^{0}=7.1 \pm 4.6 \times 10^{-21} \mathrm{~J} ; Z_{01}=1.4 \pm 0.5 \times 10^{-13} \mathrm{~N}$; $N \Delta U_{12}^{0}=-0.34 \pm 4.0 \times 10^{-21} \mathrm{~J} ; Z_{12}=2.8 \pm 1.6 \times 10^{-14} \mathrm{~N}$.

Integrity of transduction and adaptation after extreme deflections. Because the long pulse protocol involved bundle deflections of more than $1900 \mathrm{~nm}$ from the rest position, it was important to test whether these damaged transduction or adaptation. Bundles were deflected as far as they would go in the positive and negative directions by manual adjustment of the probe (Fig. $1 A-C$ ). We often held the bundle in such extreme positions for over a minute. As seen in Figure $1 C$, large negative excursions even caused stereocilia to splay as their bases came into contact. Positive excursions often pulled the kinocilium away from the bundle, but the bundle could be moved still further by repositioning the probe on the negative side of the bundle and pushing positively. In 20 out of 20 cells, no systematic diminution in the peak transduction current was detected even after several such treatments (Fig. $1 D$ ). In a few cases, rapid $(<1 \mathrm{msec})$ deflections from -2000 to $+2000 \mathrm{~nm}$ were tested, and even these failed to affect transduction. Adaptation also survived overstimulation, as seen in four out of four cells (Fig. 1E). Moreover, the extent of adaptation was unaffected; for example, it remained at $\sim 80 \%$ for the family in Figure 1 . In bundles examined ultrastructurally following overstimulation, the tip links and their dense sites of attachment to stereocilia were indistinguishable from those in control bundles (G. M. G. Shepherd, K. E. Rock, and D. P. Corey, unpublished observations).

\section{Results}

\section{Qualitative features of adaptation}

The primary features of adaptation to a sustained bundle deflection are illustrated by the step response in Figure $2 A$. At the onset of the $200 \mathrm{~nm}$ deflection, an inward current abruptly developed, which declined from its peak value back toward the baseline current level. After $150 \mathrm{msec}$, the response had diminished to about one-third of its initial amplitude. At the termination of the step, the current abruptly decreased, falling well below the baseline level before gradually returning to it. The time course of the current change has been shown to reflect shifting of the instantaneous $I(X)$ curve along the deflection axis (Corey and Hudspeth, 1983a; Eatock et al., 1987; Assad et al., 1989; Hacohen et al., 1989; Assad and Corey, 1992).
The extent of adaptation may be inferred qualitatively from such traces as the difference between the resting current level and the current remaining at the end of the step. The adapted current did not reach the initial baseline level, so the $I(X)$ curve apparently did not shift by the full extent of the deflection. However, it is not clear from this trace how complete the adaptive shift was, because in the $I(X)$ curve, receptor current is not a linear function of deflection.

The response of another cell to a family of deflections varying in amplitude is shown in Figure $2 B$. With larger positive deflections, an increasing amount of current remained at the end of the step. Negative deflections caused a decline in receptor current to the minimum value, which is probably zero (Holton and Hudspeth, 1986). Even when the current remained minimal for the duration of the step, any adaptation occurring during the step was revealed in the form of a rebound current at the end of the step, which subsequently adapted back to the resting current level. The amplitudes of the rebound transients reflect the extent to which the $I(X)$ relation shifted during the steps. Note that the termination of most negative steps produced rebound transients whose peak current levels were very similar. Adaptation for these negative steps was apparently the same, even though the step amplitude varied.

The responses shown in Figure 2 are typical of bullfrog saccular hair cells (Corey and Hudspeth, 1983a; Eatock et al., 1987; Assad et al., 1989), but there was some variability to this general pattern. Figure $2 C$ shows a cell with more resting current than that in Figure $2 B$. In this cell there was observable adaptation during negative deflections, and the rebounds indicating negative-going adaptation were not all the same. In Figure $2 D$, in contrast, there was essentially no resting current. The positive steps failed to reach saturation, suggesting that the sensitive range of the bundle was shifted rightward along the deflection axis, and the negative steps all failed to elicit much of a rebound transient at their termination. Apparently, the adaptation mech- 

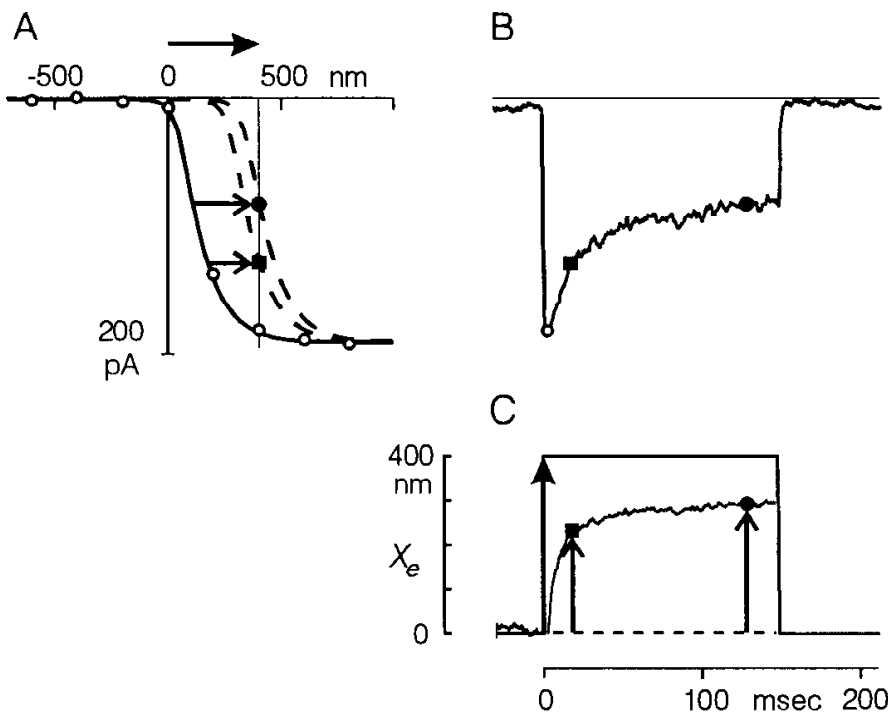

Figure 3. Inferring the adaptive shift of the $I(X)$ curve. $A$, An instantaneous $I(X)$ curve was obtained by plotting the initial receptor current $(O)$ elicited by a family of deflection steps, and fitting these points with a three-state model for channel activation (solid curve). A $150 \mathrm{msec}$ deflection of $+400 \mathrm{~nm}$ (upper arrow) was then given, eliciting the receptor current shown in $B$. For each point in the current trace, the $I(X)$ curve was shifted by the extent (lower arrows) that gave that amount of current at $+400 \mathrm{~nm}$. Two examples of shifted curves are shown as dashed lines in $A$, at $20 \mathrm{msec}(0)$ and $130 \mathrm{msec}(0)$. In $C$, the current trace was replotted as an $X_{e}(t)$ trace, showing the time course of the inferred shift. Also shown are the shifts, $X_{e}$, at $20 \mathrm{msec}(\square$ and arrow) and $130 \mathrm{msec}$ (and arrow), and the deflection stimulus, $X_{s}$ (large arrow and square wave). Current scale in $A$ and time scale in $C$ both apply to $B$.

anism encountered a limit for all negative deflections in this cell.

\section{Inferring the adaptive shift}

While these traces illustrate some interesting features of adaptation, they also demonstrate the difficulty in understanding the adaptation process on the basis of receptor current records alone. Previous studies assayed the adaptive process by measuring complete $I(X)$ curves many times during a sustained deflection, and determined the underlying mechanism of adaptation to be a shift of the $I(X)$ curve (Corey and Hudspeth, 1983a; Eatock et al., 1987; Assad et al., 1989; Hacohen et al., 1989; Assad and Corey, 1992). Such protocols can be very time consuming, however, raising concerns about the stability of the preparation and limiting the complexity of the stimuli that may be used.

To interpret the traces in Figure 2, we converted current traces, $I(t)$, into traces representing the shift of the $I(X)$ curve, $X_{e}(t)$, using a rapid method to infer the position of the $I(X)$ curve at any time (Fig. 3). First, a continuous $I(X)$ relation was obtained by fitting a three-state $I(X)$ curve (Eq. 1, with $X_{s}=X_{e}=0$ ) to the peak currents elicited by a family of deflection steps. An adapting receptor current, $I(t)$, was then mapped against the $I(X)$ curve to give the apparent shift of the $I(X)$ curve at each point in the response. In essence, Equation 1 was solved for $X_{e}$, the shift of the $I(X)$ curve (with $p_{o}$ derived from the recorded receptor current $I)$. The resulting $X_{e}(t)$ trace shows the shift of the $I(X)$ curve over time.

The inferred-shift method has the advantage of providing a continuous assessment of the $I(X)$ shift during an adapting response. It also has the advantage of speed, requiring only one burst of test pulses to map the $I(X)$ curve before an adapting step. The method works best for currents that are in the steepest portion of the $I(X)$ curve; for currents close to the minimum or maximum levels the calculated shift becomes noisy or even indeterminate. It also assumes that the value of the maximum current stays constant with time, and that the shape of the $I(X)$ curve does not change as it shifts. In agreement with previous studies (e.g., Corey and Hudspeth, 1983a; Eatock et al., 1987), these assumptions appeared to hold in the present experiments.

\section{Kinetics of inferred shift}

A family of $X_{e}(t)$ traces is shown in Figure $4 A$ for the stimulus family and original $I(t)$ traces shown in Figure $2 B$. As suggested by the original currents, the adaptation seen in the $X_{e}(t)$ traces was largely complete after 150 msec for steps of all amplitudes. The steady-state position was in all cases less than the magnitude of the step; that is, the curve shifted incompletely toward the new bundle position. Time constants for single exponential curves fitted to the traces were in the range of $15-40 \mathrm{msec}$.

To determine whether the time course depends on deflection amplitude, the $X_{e}(t)$ traces were scaled to the same stimulus size (Fig. $4 B$ ). The superposition of traces of different amplitudes suggests that the kinetics are largely independent of step size, although there was some tendency for smaller steps to have shorter time constants. A similar analysis is shown in Figure 4, $C$ and $D$, for two other cells. The completeness of adaptation, as a proportion of the deflection, was again constant for each cell, and the kinetics were roughly constant.

To examine the kinetics over much longer times, responses to individual steps lasting $1.5 \mathrm{sec}$ were analyzed in a similar way. A typical response to a positive step is shown in Figure 5; it has a brisk shift and a flat steady-state level. This behavior was seen in over 100 responses in 12 cells, for a wide variety of step amplitudes.

We attempted to fit the time course of the $I(X)$ shift with exponential curves. Many traces could not be fitted, because steps that opened or closed most of the channels produced saturation or excessive noise in the $X_{e}(t)$ traces. Of the fitted traces, most were adequately described by a single exponential. In some cells, a better fit was obtained by summing two exponential time constants of 6-10 msec and $100-120 \mathrm{msec}$. There was no evidence of a relaxation with a time constant longer than $300 \mathrm{msec}$, although a very slow component of adaptation is not ruled out by these experiments. The average time constant for all the single-exponential fits was $25 \pm 12 \mathrm{msec}( \pm \mathrm{SD} ; N=126$ traces; range, 6-65 $\mathrm{msec}$ ). Time constants for positive- and negativegoing steps were not significantly different.

\section{Extent of adaptation measured by inferred shift}

The foregoing kinetic analysis indicated that adaptation proceeded to its final extent within $150 \mathrm{msec}$; for an average time constant of $25 \mathrm{msec}$, the shift was within $0.3 \%$ of its final value at that time. We therefore measured the extent of adaptation at $150 \mathrm{msec}$ using the inferred-shift method. From a total of 41 stimulus families measured in 17 cells, we chose nine families from eight cells according to the analysis criteria (see Materials and Methods, Analysis of current traces). Data were analyzed essentially as described in Figure 3, except that current was averaged before comparison to the resting $I(X)$ curve to determine the shifted position of the curve: for positive deflections, currents were averaged at steady state, between 130 and 150 msec; for negative deflections, the peak of the rebound current 

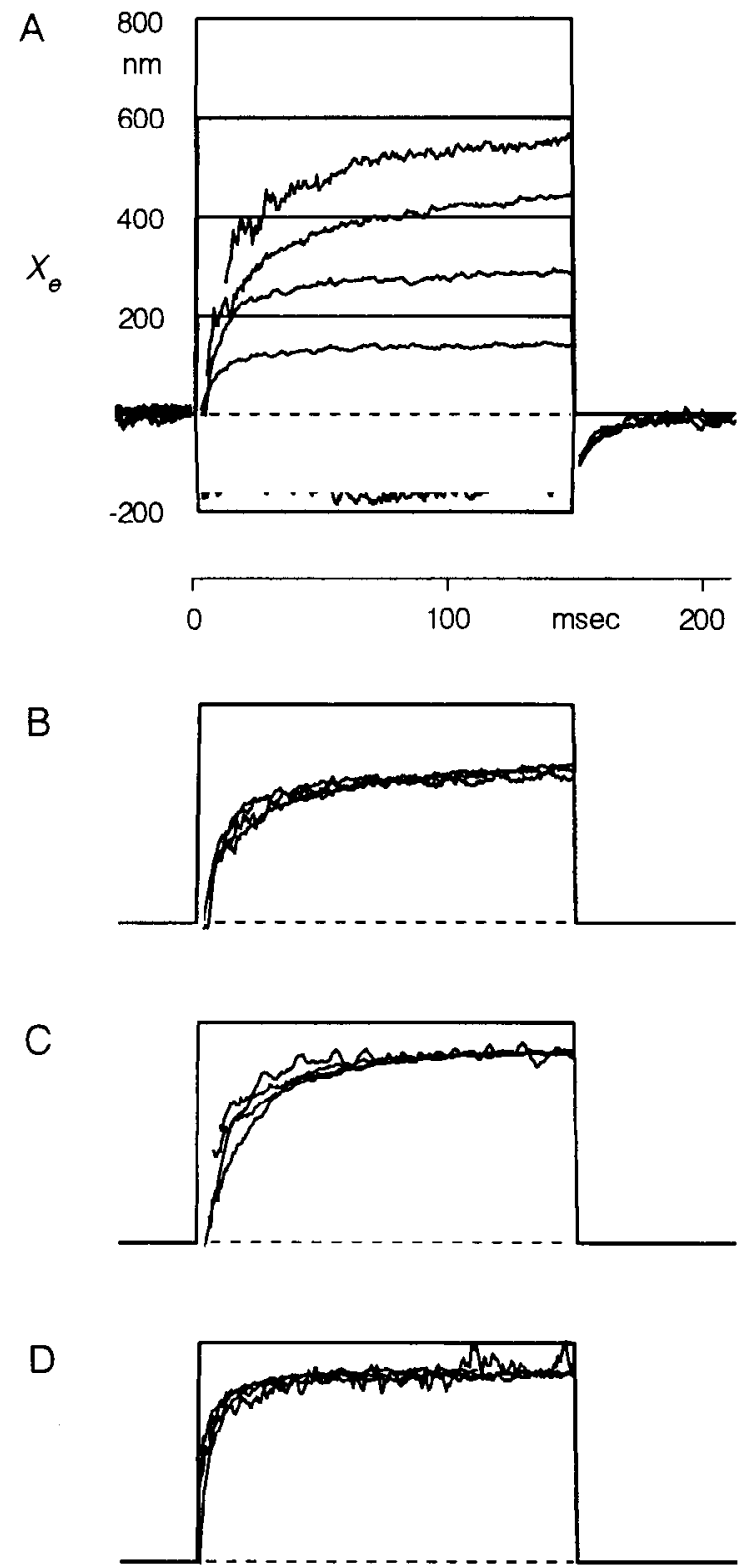

Figure 4. Kinetic features of adaptation. A, Deflection stimuli (square waves) and $X_{e}(t)$ traces are shown for deflections of $+800,+600,+400$, +200 , and $-200 \mathrm{~nm}$; traces are also drawn for just the initial and rebound responses to deflections of -400 and $-600 \mathrm{~nm}$. For all positive deflections the $I(X)$ curves shifted incompletely toward the new bundle position. During deflection to $-200 \mathrm{~nm}, X_{c}$ was mostly indeterminate, due to saturation of the $I(X)$ curve (gaps in trace). The rebounds following negative deflections shared a common amplitude and time course. $B$, The $X_{e}(t)$ traces in $A$ were scaled to a common deflection amplitude, and appear to be scaled versions of each other. Examples from two different cells are shown in $C$ and $D$, representing the range of responses observed.

was measured by averaging over about $2 \mathrm{msec}$ following the termination of the step.

The extents of the $I(X)$ shifts $X_{e}$ were plotted against the deflection amplitudes $X_{s}$; one example is shown in Figure 6A. The proportional extent of adaptation is the ratio $X_{e} / X_{s}$. The dashed line indicates complete adaptation - the line expected if the shift of the $I(X)$ curve is exactly equal to the deflection, giving a proportional extent of 1.0. The data fall into two groups. First, over the range of bundle positions between $+800 \mathrm{~nm}$ and about $-200 \mathrm{~nm}$, the points appear to follow a straight line whose
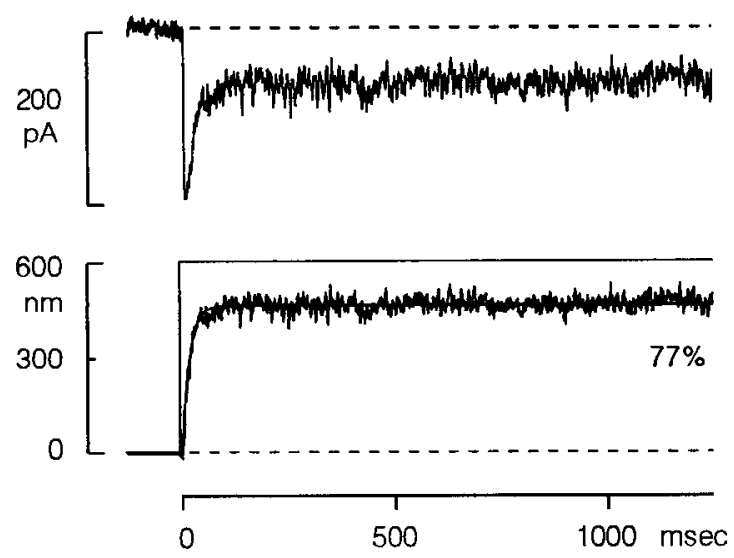

Figure 5. Adaptive response to a long step deflection. Upper trace shows the current elicited by a $+600 \mathrm{~nm}$ step lasting $1.5 \mathrm{sec}$. Lower trace shows the calculated $X_{e}(t)$ trace and stimulus. The trace was fitted with a single exponential curve $(\tau=16 \mathrm{msec})$. Within $150 \mathrm{msec}$, the shift reached a stable plateau at $77 \%$ of the stimulus amplitude.

slope is slightly below unity; the slope of a line fitted to these points (not shown) was 0.82 . Second, the points negative to a bundle position of about $-200 \mathrm{~nm}$ in this cell fall along a straight line whose slope is close to zero.

The responses of all nine families are shown superimposed in the plot in Figure $6 B$. Again, the data fell into two regions, one with a slope just below unity, the other with a slope close to zero. The plots differed somewhat in the position of the transition point between the two regions. Since the absolute zero position is not well defined, we normalized the bundle position to this transition area (see Fig. 6 legend).

The normalized plots for all families are shown in Figure $6 C$. The slope of the positive-side best-fit line was 0.79 , while that of the negative-side line was 0.05 . That is, adaptation proceeded with $79 \%$ and $5 \%$ efficiency to the right and left, respectively, of the transition point. The average $X$ value of the transition point was $-145 \pm 68 \mathrm{~nm}$. Because little or no adaptation occurred beyond the transition point, it appeared to constitute a negative limit for the adaptation mechanism.

\section{Extent of adaptation measured by $\mathrm{I}(\mathrm{X})$-curve comparison}

The assumption that adaptation plateaus within $150 \mathrm{msec}$ was based on the short time constant $(25 \mathrm{msec})$ of the initial phase of adaptation. The $X_{e}(t)$ traces inferred from the responses to $1.5 \mathrm{sec}$ deflections (Fig. 5) also indicate little if any slow component of adaptation. However, the inferred-shift method is indirect, and we considered it important to check more directly for slow components and to obtain a measure of the extent of adaptation by an independent method.

The adaptive shift of the $I(X)$ relation was measured directly by comparing the positions of instantaneous $I(X)$ relations sampled before and during long adapting steps. We used an adapting step pattern based on multiples of $100 \mathrm{~nm}$ steps, maintained for $4.0 \mathrm{sec}$ (Fig. $7 A$ ). A resting $I(X)$ relation was sampled just before each step. Another $I(X)$ relation was sampled after 2 sec. Resting instantaneous $I(X)$ points were fitted with the activation curve of Equation 1 (with $X_{e}=0$ ); $X_{e}$ was then varied to fit the shifted $I(X)$ points. Unlike the inferred-shift method, this method was effective in fitting positive and negative $I(X)$-curve shifts of any magnitude (Fig. $7 B, C$ ). The $X_{e}$ values obtained in this way were plotted against the amplitude of the deflection $X_{s}$ (Fig. $7 D$ ). Had the shifts been complete, the points would have fallen 

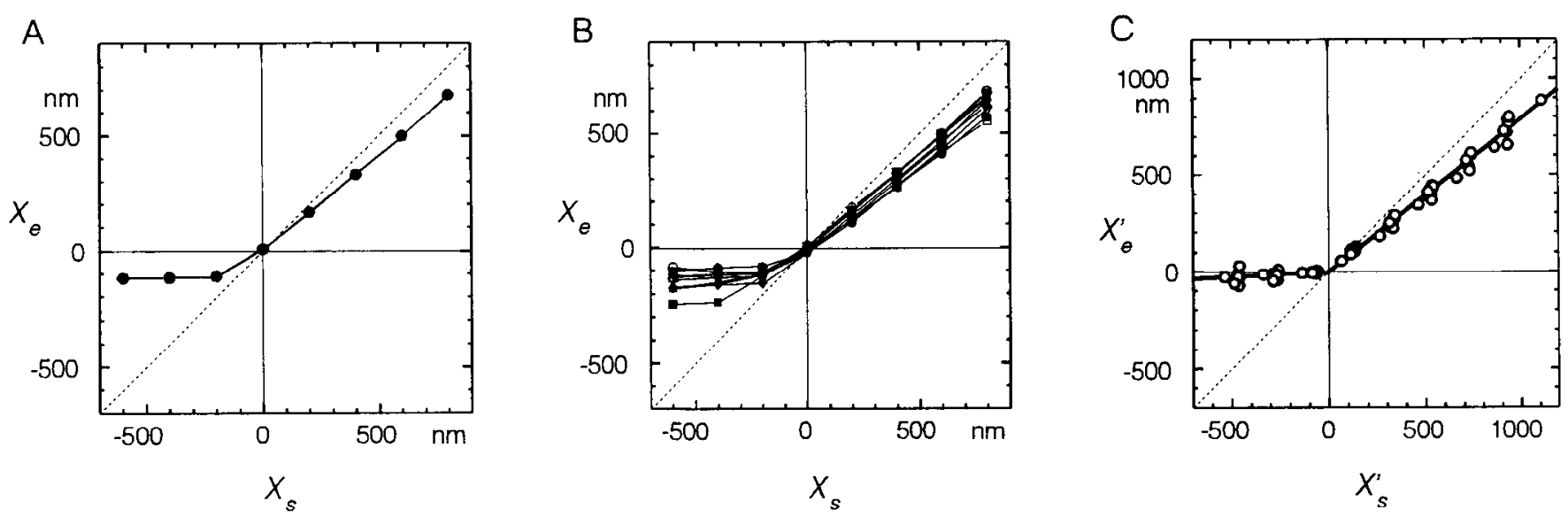

Figure 6. Extent of adaptation at $150 \mathrm{msec}$, measured by the inferred-shift method. $A$, For a family of deflections, $X_{e}$, the adaptive shift at 150 msec was plotted against $X_{s}$, the deflection amplitude. The dashed line represents complete (100\%) adaptation. For positive deflections, $X_{\text {, }}$ appears to be in constant proportion to $X_{s}$. For larger negative deflections, adaptation proceeded only up to a distinct limit. $B$, The subunity slope in the positive direction and the limit in the negative direction were consistent features for all cells, as shown by superimposing data for nine families. $C$. To compare different cells with different negative limits, the data were normalized as follows. For each family, lines were fitted by least-squares minimization to data points on either side of the position of the apparent negative limit; the process was reiterated for other positions to find that which minimized the total sum of the two fits. The intersection of the fitted lines was calculated, and its $x$ - and $y$-values subtracted from all the data as a way of normalizing them to a common origin. Best-fit lines have slopes of 0.79 in the positive region and 0.05 in the negative region.

along the unity slope line, as before. Figure $7 D$ shows a typical run, in which the slope fell slightly below unity for positive deflections, and a definite negative limit occurred at $-333 \mathrm{~nm}$. In this cell, the extent appeared to saturate for the largest positive deflections as well. Although such data initially led us to speculate that there was also a positive limit (Shepherd et al., 1991), this was not a consistent finding; rather, the average extent was linear in the positive direction.

From a total of 71 families in 44 cells, 15 families from 11 cells met the analysis criteria. Four families are shown superimposed in Figure $7 E$. The families show most of the same features of the extent seen at short times, including a positive region with near-unity slope, and a negative region with nearzero slope. The position of the negative limit differs substantially among cells, however.

To compare the plots from different cells and bundle positions we used the same normalization technique employed with the short-duration families. All 267 data points thus derived are plotted in Figure $7 F$. Several key features are evident. The transition zone, at the origin, is strikingly sharp. The slope of the line fitting all positive points was 0.83 . Slopes measured for smaller distances ( 0 to $+400 \mathrm{~nm}$ ) varied by only a few percent, indicating that there was no apparent saturation for positivegoing adaptation. The slope of the line fitting all negative points was 0.14 , but was close to zero for many of the individual families. Four cells were stable enough for two or more longduration families to be recorded. These, too, gave extent plots that closely resembled each other both in the slopes of the two regions and in the point of intersection (the negative limit). In several cells, both the short- and long-duration protocols were tested. The extent plots generated by either method all resembled each other closely.

In some particularly stable cells, we were able to collect complete data sets for the long-duration family at several different resting bundle positions, by manually giving a static bias to the bundle between families. In such cases, the slopes of the two regions remained the same, but the negative limit (relative to the resting position) was shifted by the amount of the static bias.
The cell in Figure $7 E$ (square) that appears to have no negative limit had been given a $+1000 \mathrm{~nm}$ static bias just prior to testing. When it was tested at more negative resting positions, the negative limit was observed. This illustrates that the negative limit is fixed in position, relative to the absolute bundle position; it does not move with static bundle deflections, at least over a time of minutes. It also indicates that the cxtent of adaptation is a linear function of deflection over a very large range, at least up to $+2000 \mathrm{~nm}$.

In some cells, the peak amplitude of the transduction current appeared to diminish by $10-20 \%$ with the largest positive deflections, although this was not a very consistent effect. Consequently, we fitted the shifted $I(X)$ curves in two ways: either allowing the amplitude to vary or not. There was no significant difference between the two methods in the measured extent of adaptation.

\section{Steady-state $\mathrm{I}(\mathrm{X})$ curves}

The basis of incomplete adaptation is a shift of the $I(X)$ curve that is less than the sustained deflection of the bundle. Its most obvious manifestation is a transduction current that fails to return to the resting value at steady state. For the families of 150 msce deflections, we plottcd the stcady-statc current, obtained by averaging the last $\sim 20 \mathrm{msec}$ in the steps, against the deflection to generate "steady-state $I(X)$ curves." These are shown in Figure 8. For comparison, we also plotted the instantaneous $I(X)$ curve, derived from the peak instantaneous currents. If the extent of adaptation is a simple linear function of the amplitude of the adapting step, as the data of Figures 6 and 7 strongly suggest, then the steady-state $I(X)$ should be identical to the instantaneous $I(X)$ relation, scaled in proportion to the extent of adaptation. The scaling is equivalent to "stretching" the instantaneous $I(X)$ curve around the zero-deflection position, by a factor of $1 /\left(1-X_{e} / X_{s}\right)$, where $X_{e} / X_{s}$ is the proportional extent of adaptation.

Typical examples of steady-state and instantaneous $I(X)$ relations are presented in Figure $8 A-C$. The extents of adaptation, measured as the value of $X_{e} / X_{s}$ providing the best least-squares 
A

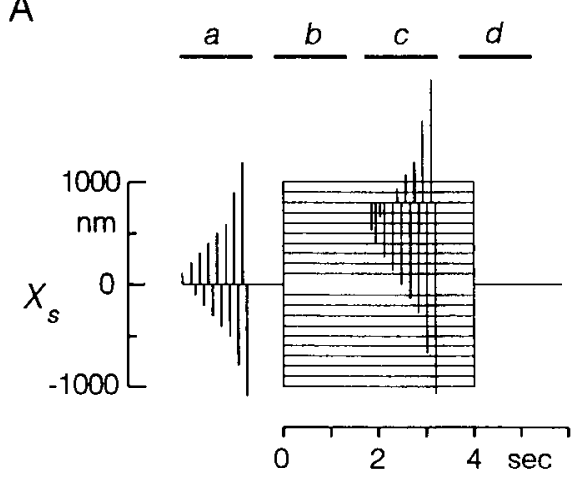

D

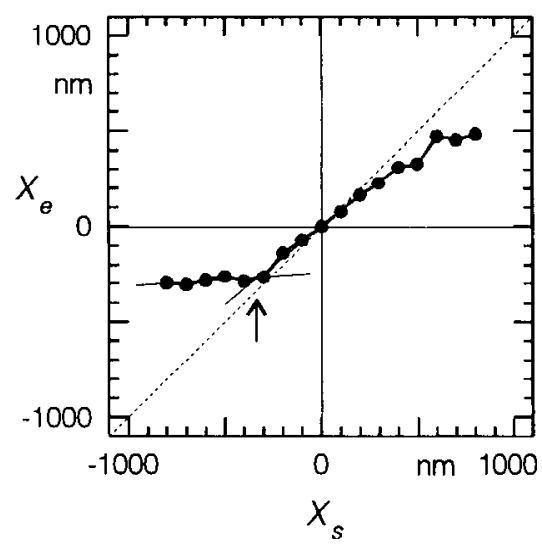

B

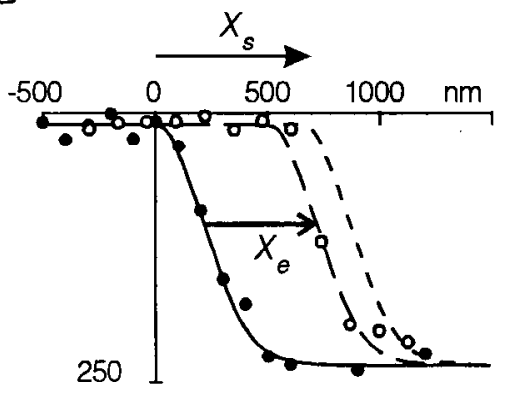

pA

E

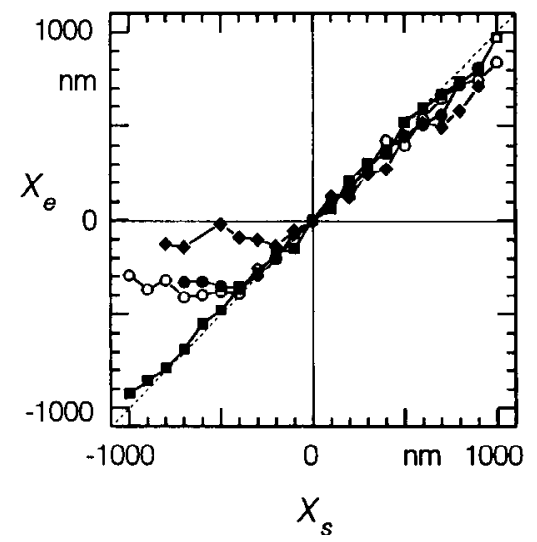

C

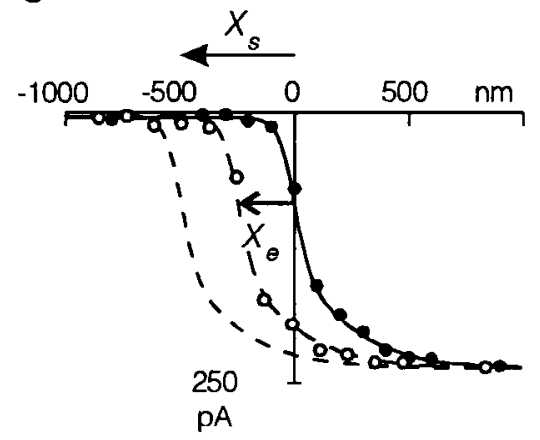

F

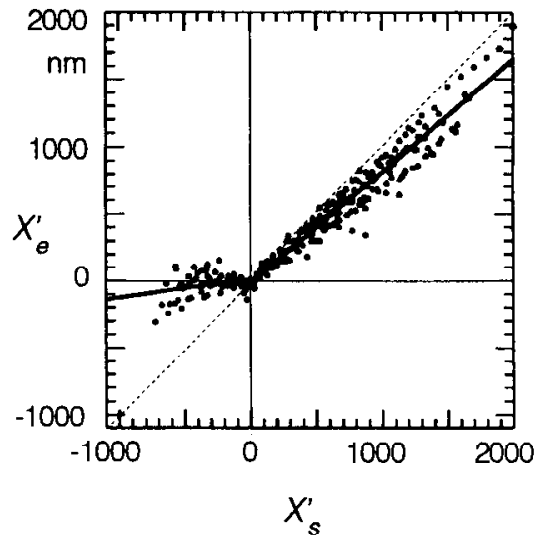

Figure 7. Extent of adaptation after $2.0 \mathrm{sec}$, measured by comparing $I(X)$ curves before and during deflections. $A$, The stimulus protocol (see Materials and Methods) started with a burst of test deflections to obtain an $I(X)$ curve at the resting position $(a)$. The bundle was next deflected, and receptor current recorded for $1.5 \mathrm{sec}(b)$. Another burst was delivered to obtain the $I(X)$ curve in the adapted state $(c$; only one burst pattern drawn, for the $+800 \mathrm{~nm}$ step). The rebound response generated as the step was terminated was recorded (d). The cycle was repeated until all deflections up to $\pm 1000 \mathrm{~nm}$ were tested. $B$. The extent of adaptation was measured by fitting Equation 1 to the initial $I(X)$ data $(O)$, then shifting it to fit it to the adapted $I(X)$ data $(O)$. The example shows a deflection, $X_{s}$, of $+700 \mathrm{~nm}$ and a shift, $X_{e}$, of $+500 \mathrm{~nm}$. The actual shifted curve (long dashes) may be compared with a 100\% shifted curve (short dashes). $C$, Negative-going adaptation was evaluated by the same method. The example shows a deflection, $X_{s}$, of $-500 \mathrm{~nm}$ and a shift, $X_{e}$, of $-245 \mathrm{~nm}$. A $100 \%$ shifted curve is shown for comparison. $D$, For a family of deflections, the adaptive shift at $2 \mathrm{sec}, X_{e}$, was plotted against the deflection amplitude, $X_{s}$. The points occur in two regions, one in which adaptation proceeded fairly efficiently (positive to arrow), and one in which adaptation was almost completely absent (negative to arrow). Lines represent leastsquare fits to the first five data points to either side of the arrow, which points to the intersection of the lines. $E$, Data from four cells are shown, indicating the variability in the negative limit. $F$, Fifteen plots from 11 cells are shown, normalized as in Figure 6 by aligning the intercepts. The 263 points segregate into two regions to either side of zero. Best-fit lines have slopes of 0.83 in the positive region and 0.14 in the negative region.

fit to the steady-state $I(X)$ data, were comparable to the extents measured by the inferred-shift method. The curves were well fitted by the stretched instantaneous $I(X)$ curves, and only slightly better fits were achieved by allowing $N \Delta U_{01}^{0}, Z_{01}, N \Delta U_{12}^{0}$, and $Z_{12}$ in Equation 1 to vary independently. Among seven families that were analyzed, the extent of adaptation measured by fitting the steady-state $I(X)$ curves varied from $69 \%$ to $90 \%$, with an average extent of $83 \%$. This agreed closely with the average value of $79 \%$ obtained from the inferred-shift method.

Similar plots (Fig. $8 D-F$ ) were generated for the responses measured about $1.5 \mathrm{sec}$ after adapting steps in the $4.0 \mathrm{sec}$ deflection protocol (at the end of interval $b$ in the protocol shown in Fig. $7 A$ ). Data were noisier because there was no averaging of traces, but the larger number and wider range of deflections provided a broader measurement of the steady-state current. The data shown represent typical fits and extents. The extents measured at $1.5 \mathrm{sec}$ by fitting the steady-state $I(X)$ curve were comparable to the extents measured with the same protocol by curve comparison. The proportional extents for 10 families an- alyzed with several different resting positions varied from $60 \%$ to $93 \%$, with most values falling between $76 \%$ and $84 \%$.

Thus, a cell's sensitivity appears to be characterized by both an instantaneous and a steady-state $I(X)$ relation, where the steady-state relation is roughly fivefold less sensitive but the response range is fivefold broader.

\section{"Resting" transduction current}

An implication of the steady-state $I(X)$ curve is that the proportion of the transduction current that is activated at rest depends on the static bundle position. This complicates the interpretation of experiments that use direct coupling of a stimulus probe, since there is unavoidable deflection of the bundle in the process of attaching the probe and there is subsequent drift in the bundle position. The data in Figure 8 indicate that a static bias of only a few hundred nanometers can change the resting current substantially due to the incompleteness of adaptation. Unfortunately, even with high-resolution video observation, it can be hard to detect a drift of this amount. Moreover, the angle 
Figure 8. Extent of adaptation at 150 msec and $1.5 \mathrm{sec}$ measured from steadystate $I(X)$ curves. Instantaneous $I(X)$ currents $(\bullet)$ were fitted with Equation 1 , and steady-state $I(X)$ currents $(O)$ were fitted with the same curve stretched along the $x$-axis. Currents were measured $150 \mathrm{msec}$ after deflections of -600 to $+800 \mathrm{~nm}(A-C)$, or $1.5 \mathrm{sec}$ after deflections up to $\pm 1000 \mathrm{~nm}(D-F)$. The extent of adaptation measured by stretching the instantaneous $l(X)$ curve is shown as a percentage of complete adaptation. Shown for comparison are extents (parentheses) and negative limits to adaptation (arrows) measured by inferring $(A-C)$ or directly measuring $(D-F)$ the $I(X)$ shift.
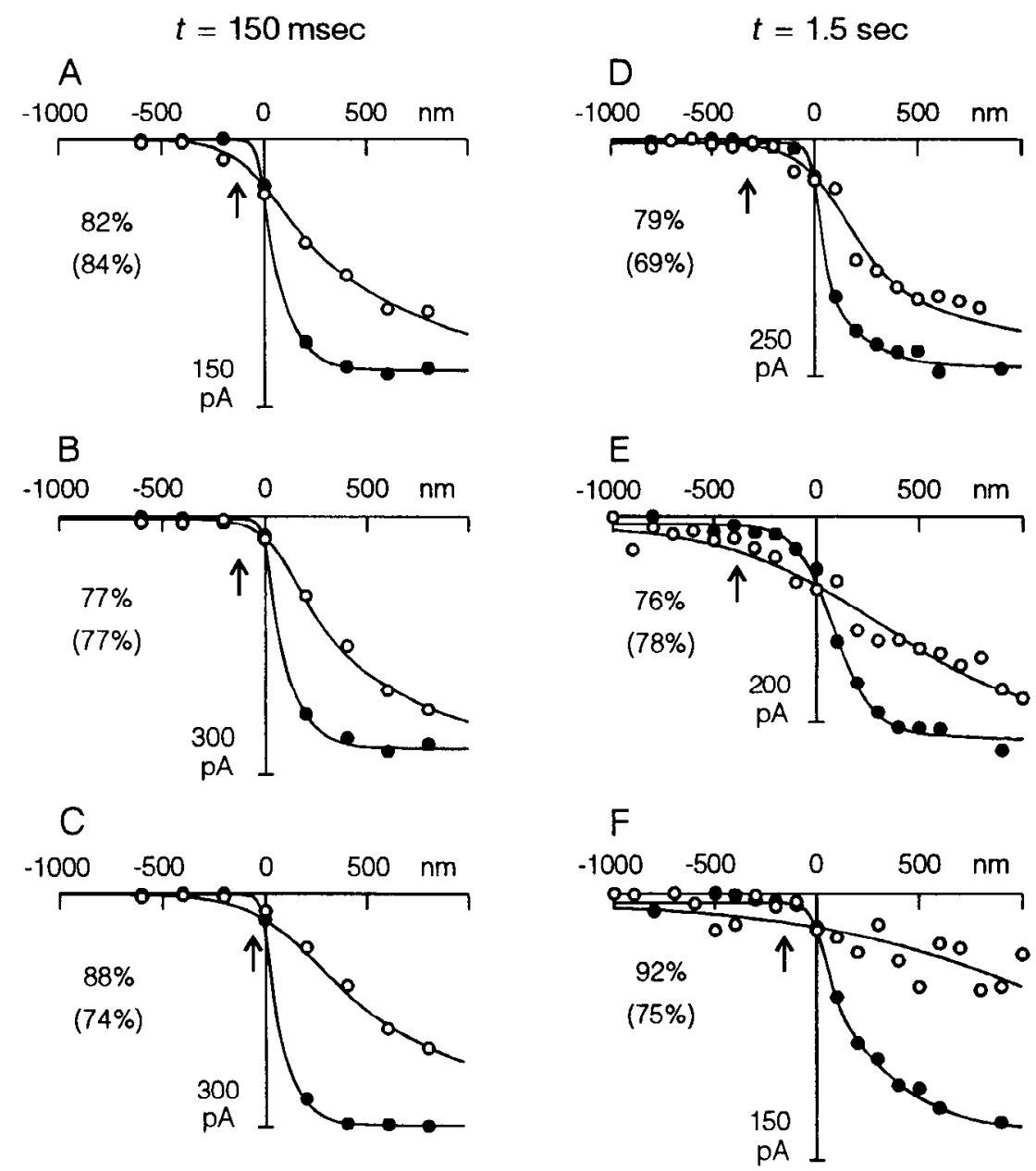

of the bundle relative to the cell body does not offer a reference, since it varies considerably among cells even within the same epithelium. For example, Figure 9 shows a cell whose hair bundle was given static deflections of $-900,0$, and $+900 \mathrm{~nm}$; for each "resting" bundle position the corresponding instantaneous $I(X)$ curve is also shown. The proportion of transduction current active at rest varies between $0 \%$ at $-900 \mathrm{~nm}, 15 \%$ at $0 \mathrm{~nm}$, and $68 \%$ at +900 , yet all three bundle positions would be considered within the normal range for unrestrained bullfrog saccular hair cells. Note the limited negative-going shift, due to the negative limit.

Since the bundle position does not provide an absolute indication of the resting current level, we measured the "true" resting current level in freestanding hair bundles by deflecting them with a fluid-jet stimulator. While the transduction current was recorded with whole-cell patch clamp, a puffer pipette was brought up to the bundle, a series of saturating positive and negative fluid-jet stimuli were delivered, and then the pipette was removed. In 14 cells measured in extracellular solution containing $4 \mathrm{mM} \mathrm{Ca}^{2+}$, the average resting current was $9 \pm 4 \%$ $( \pm \mathrm{SD})$ of the maximum. This value was similar to that obtained with a mechanical probe when care was taken in coupling it to the bundle.

\section{Discussion}

We have measured the extent of adaptation in three ways: (1) receptor currents before and after adaptation were compared using the resting instantaneous $I(X)$ curve to infer the adaptive shift, (2) instantaneous $I(X)$ curves obtained before and after adaptation were compared to give a more direct measure of the shift, and (3) instantaneous $I(X)$ curves were stretched to fit steady-state $I(X)$ currents, the magnitude of stretch indicating the extent of adaptation. In each case, the adaptive shift of the instantaneous $I(X)$ curve was a constant proportion of the deflection, for deflections positive to the negative limit.

\section{Possible artifacts}

We considered whether the limited extent of adaptation could be an artifact. One possibility is that the cells were inadequately attached to the substrate, since any movements of the cell bodies during deflections would be interpreted as shifts of the $I(X)$ curve. However, we took great pains to study only cells that showed no movement on a high-magnification video monitor. More importantly, movement would tend to make the measured adaptation more rather than less complete.

Another possibility, that the cells were unhealthy and unable to adapt normally, may be ruled out for several reasons. The cells were morphologically normal and survived for as long as several hours in a bath solution that was well oxygenated and refreshed frequently. Moreover, the limited adaptation and negative limit were seen both early and late in an experiment, and in all cells. The cells whose adaptation was studied all had robust transduction currents $(100-400 \mathrm{pA})$. Within that range, there was no particular correlation of current magnitude and the ex- 

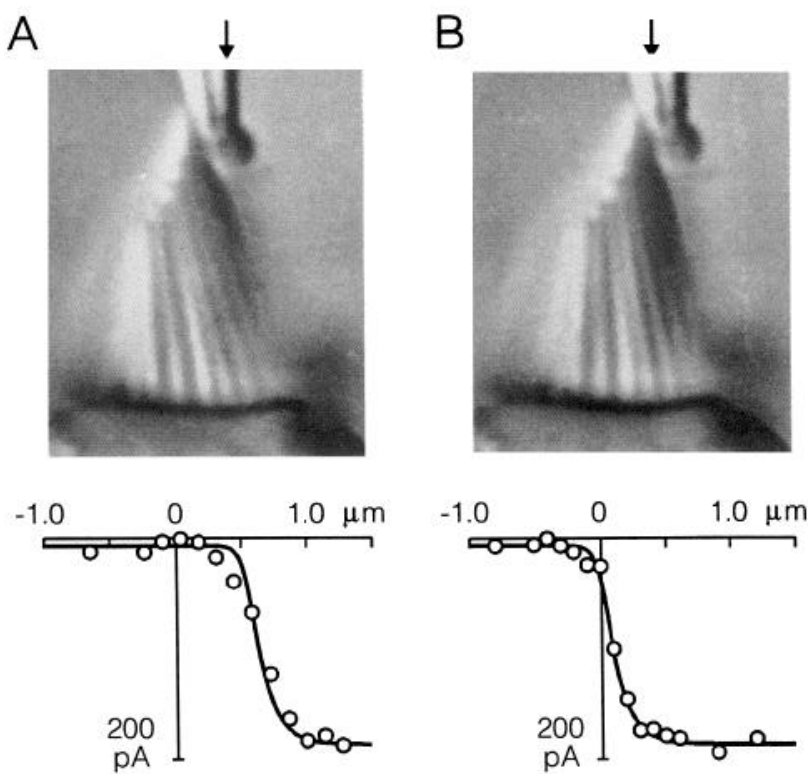

tent of adaptation. Even in cells disqualified from further analysis due to cell-body rocking or small transduction currents, the limited extent of adaptation was qualitatively similar.

One might expect more variation in the extent of adaptation among different bundles and at different times during an experiment if incompleteness is an impairment of normal function. The limited adaptation was not due to a lack of ATP, which was present in the internal solution. Besides, the exchange of ADP $\beta$ S for ATP arrests adaptation entirely (Gillespie and Hudspeth, 1993). Under pathological conditions, one might expect the kinetics to be hindered more than the final extent, but the kinetics were comparable to those of receptor currents recorded previously in different preparations, such as the more intact saccular microphonic preparation (Eatock et al., 1987). Not surprisingly, the same limited extent of adaptation was seen in cells with or without a kinocilium (data not shown); neither transduction (Hudspeth and Jacobs, 1979) nor adaptation (Assad and Corey, 1992) requires an intact kinocilium.

\section{Resilience of mechanosensitivity}

The incompleteness of adaptation observed in these studies was also not an artifact induced by the large deflections used in the stimulus protocols, since transduction and adaptation were unaffected by even the largest stimuli. Conversely, extreme deflections neither induced nor changed the magnitude of the incompleteness of adaptation. That transduction survived extreme mechanical distortions is perhaps surprising, given the reknowned sensitivity of the bundle. If the tip links are the gating springs that convey tension to the mechanical transduction channels (Pickles et al., 1984; Assad et al., 1991), then they apparently tolerate stretching to at least twofold their resting length. For example, the largest stimuli used to push bundles in the positive direction were well over $5000 \mathrm{~nm}$ (and well over $45^{\circ}$ of angular rotation around the basal insertions of stereocilia). An extent of adaptation of $80 \%$ implies an effective steady-state stimulus of over $1000 \mathrm{~nm}$; the instantaneous stimulus was even larger. Taking into account the geometry factor of $0.12-0.14$, which relates bundle position to shear along the sides of stereocilia (Howard and Hudspeth, 1988; Corey et al., 1989), this amounts to at least $120 \mathrm{~nm}$ of stretch of the tip link, beyond its resting length of $\sim 150 \mathrm{~nm}$ (bullfrog saccular hair cells; Jacobs
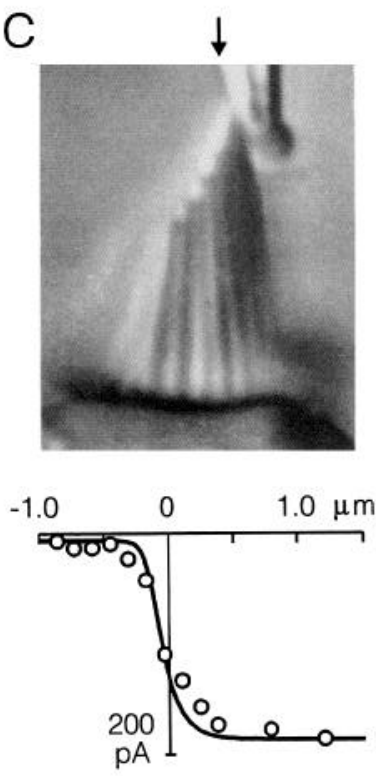

Figure 9. Dependence of the "resting" $I(X)$ curve on bundle position. A dissociated hair cell's bundle was held at its freestanding position, and an $I(X)$ relation was measured with a family of short deflections. The bundle was then given static deflections that were still within the "normal" range of bundle positions, and after several seconds a new instantaneous $I(X)$ curve was measured at the offset positions. $A-C$ show static deflections of $-900,0$, and +900 $\mathrm{nm}$ and the corresponding instantaneous $I(X)$ relations ( fiduciary arrows mark the same $x$-position). A curve was fitted to the data at the nominal resting position in $B$. A shift of $-360 \mathrm{~nm}(40 \%)$ provided the best fit to the data in $A$, and a shift of $+746 \mathrm{~nm}(83 \%)$ provided a best fit to the data in $C$. and Hudspeth, 1990; Shepherd et al., 1991). In a few cells, transduction current also survived fast deflections to a position of $+2000 \mathrm{~nm}$, which stretched tip links by at least $240 \mathrm{~nm}$. Failure to destroy mechanosensitivity by extreme mechanical deformations is consistent with structural and functional studies of sound-induced damage in cochlear hair cells, in which the loss of tip links was seen only in disrupted bundles (Pickles et al., 1987), and impairment of auditory responses was associated only with abnormal bundle morphologies and damage to the stereociliary rootlets in particular (Liberman, 1987).

\section{Homogeneity of components}

A single cell contains 50 or so transduction units (tip link, one or more channels, and motor). Do all units fail to adapt by the same proportion, or does some subset fail to adapt at all while the rest adapt completely? In the latter case, adapted $I(X)$ curves would be the sum of a fully adapted and a nonadapted component, and the shape would be broader than the resting $I(X)$ curve. While slight broadening is sometimes seen, it is far less than would be expected from a heterogeneous population of adaptation units. Also, a nonadapting component does not explain the negative limit, beyond which no part of the $I(X)$ curve adapts. It is most parsimonious to assume a homogeneous population.

\section{Functional significance of limited extent}

The generation of the steady-state $I(X)$ relation is illustrated in Figure $10 A$, which shows the shift of the instantaneous $I(X)$ curve (bold) in response to deflections incremented by $500 \mathrm{~nm}$. Shifted $I(X)$ curves are shown for a proportional extent of $80 \%$, and the steady-state current at each deflection is indicated by an open circle. The steady-state $I(X)$ curve, shown by a dashed line, is a fivefold broader version of the instantaneous curve. Figure $10 B$ shows steady-state (X) curves calculated with different proportional extents. For $100 \%$ adaptation, the instantaneous curve shifts completely, giving a steady-state curve which is simply flat and a resting current which is constant and deflection independent. For $25 \%$ or $50 \%$ adaptation, the shift of the instantaneous $I(X)$ curve is substantial, yet the steady-state curve appears very similar to the instantaneous curve, and the resting current is near saturation for all but the smallest deflec- 

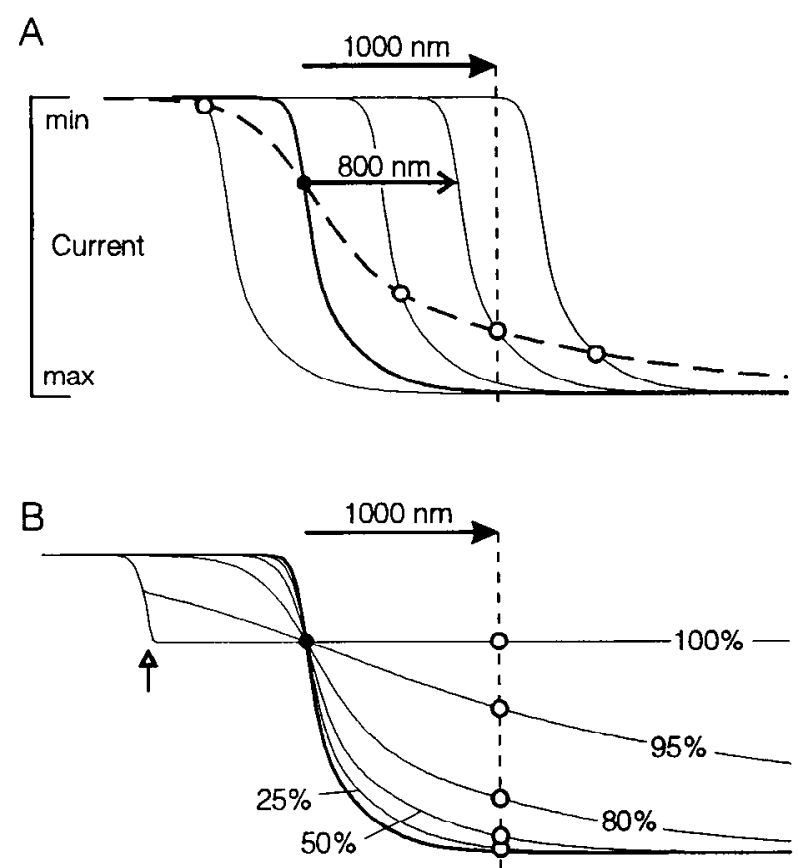

Figure 10. Relationships of instantaneous and steady-state $I(X)$ curves. $A$, The resting instantaneous $I(X)$ curve $($ bold $)$ has a resting current level at the zero bundle position indicated by the solid circle. Shifts of the instantaneous curve are shown for $80 \%$ extensive adaptation to deflections incremented by $500 \mathrm{~nm}$. The arrows indicate an $800 \mathrm{~nm}$ shift for a $1000 \mathrm{~nm}$ deflection. The steady-state currents after adaptation $(O)$ form the steady-state $I(X)$ curve (dashed), which is a fivefold stretched version of the instantaneous curve (see Discussion). $B$, Steady-state $I(X)$ curves are drawn for different values of the extent of adaptation. Again, the solid circle indicates initial resting current, and open circles indicate the steady-state currents. For the $1000 \mathrm{~nm}$ deflection shown, the steadystate current is very sensitive to changes in extent from about $50-95 \%$. In the negative direction, adaptation encounters a negative limit (open arrow). For $>90 \%$ adaptation, further negative deflection would be expected to cause a decline in current to zero with the shape of the instantaneous $I(X)$ curve. Experimentally, the negative limit was always at a steady-state current level close to zero, and therefore this effect would not have been apparent in the steady-state curve.

tions. Thus, the extent of adaptation must be in the $70-100 \%$ range for adaptation to be very obvious with simple stimulus protocols.

The major consequence of the limited extent is that the adaptive response seen at the level of the receptor current is clearly phasic-tonic in character: the current during a maintained deflection shows a large initial change declining to a smaller steadystate value. Such a response contains information about both the rate of change and the final amplitude of the stimulus. It is interesting that the nonlinear nature of the $I(X)$ curve causes the level of the tonic response to depend on the size of the stimulus. For small stimuli within the linear range of the $I(X)$ curve, the tonic value will be $(1-0.8)=0.2$ times the peak. However, for larger stimuli, the phasic response is saturated while the tonic response is not, making the tonic portion proportionally larger. This can be seen in Figure 10, where the tonic response to a $+1000 \mathrm{~nm}$ stimulus, even for $80 \%$ complete adaptation, is more than half the phasic component. For the largest stimuli, both phasic and tonic components are saturated andeven though the $I(X)$ curve shifts by $80 \%$ - there is no observable adaptation of the receptor current.

Adaptation resembling that recorded in bullfrog sacculus oc- curs in hair cells from turtle basilar papilla (Crawford et al., 1989) and mammalian cochlea (Kros et al., 1992). In both, adaptation of receptor currents is clearly incomplete. Among the four types of bullfrog utricular hair cells, adaptation of receptor potentials varies considerably in extent (Baird, 1992). In goldfish sacculus, adaptation of microphonic potentials is apparently limited, although the magnitude of the instantaneous response is unclear, due to the slow onset of the sinusoidal stimulus (Furukawa and Matsuura, 1978). In otolith organs from a variety of species, adaptation seen in afferent fiber responses often spares a tonic component (e.g., Lewis et al., 1982; Goldberg and Fernández, 1984; Baird and Lewis, 1986; Eatock et al., 1987). Regardless of where the primary adaptation process occurs in hair cell sensory pathways, any nonadapting components must originate in the bundle as incomplete adaptation of the transduction current.

\section{Revised model for adaptation}

A model for the adaptation process was previously developed based on an active motor element that generates tension in the gating spring (Assad and Corey, 1992). This motor was characterized by a zero-tension climbing rate, $C$, and a tensiondependent slipping rate, $S X_{g} . S$ and $C$ were derived from the initial rates of adaptation, measured in the first few milliseconds following a bundle deflection; $X_{g}$ is the stretch of the gating spring. Resting tension in the gating spring was determined by the balance between slipping and climbing, as suggested by Howard and Hudspeth (1987). The model successfully predicted a shift of the $I(X)$ curve with voltage, and voltage-dependent movement of freestanding hair bundles.

The model assumes that adaptation is complete, faithfully restoring $X_{g}$ and $p_{o}$ to their resting values following deflections of any magnitude. While the observations in this paper do not invalidate the conclusions of Assad and Corey, it is clear that the model is insufficient. To account for our new results-specifically, the incompleteness of adaptation and the negative limit - two new elements must be added to the mechanical model (Fig. 11). The incompleteness requires an extent spring, $\mathrm{K}_{e}$, in parallel with the motor. The stiffness of the extent spring is some fraction, $\phi$, of that of the gating spring: $\mathrm{K}_{e}=\phi \mathrm{K}_{g}$. Thus, if $\phi=$ 0.25 , the proportional extent of adaptation is $1 /(1+\phi)=0.8$. The negative limit, a deflection beyond which adaptation does not occur, requires a stop. This is characterized by a distance, $X_{n}$, such that shortening of the extent spring $X_{e}$ cannot be greater in magnitude than $X_{n l}$. In these experiments, $X_{n l}$ is typically -100 to $-500 \mathrm{~nm}$, and the stop is indicated by setting $X_{e} \geq$ $X_{n /}$. In addition, balancing the new force terms added by these elements requires that we define the motor more specifically. The "black-box" motor previously characterized by the parameters $C$ and $S$ is now recast as a force source, $F_{m}$, in parallel with a dashpot, $\Xi_{m}$. The number of free parameters remains the same, and the new parameters, $F_{m}$ and $\Xi_{m}$, can be defined in terms of the old $C$ and $S$. Also, this characterization suggests comparison to motile systems that are characterized in terms of an isometric force, $F_{m}$, and a zero-tension velocity, $F_{m} / \Xi_{m}$ (Hill, 1938). Lastly, a minor change from the previous model is that the motor position is specified as the stretch of the extent spring, $X_{c}$, rather than an arbitrary $X_{m}$, so that slipping in response to positive bundle deflection, which causes a positive shift of the $I(X)$ relation, results in a positive change in $X_{e}$, in contrast to the negative change in $X_{m}$. Details of the revised model are presented in the Appendix. 


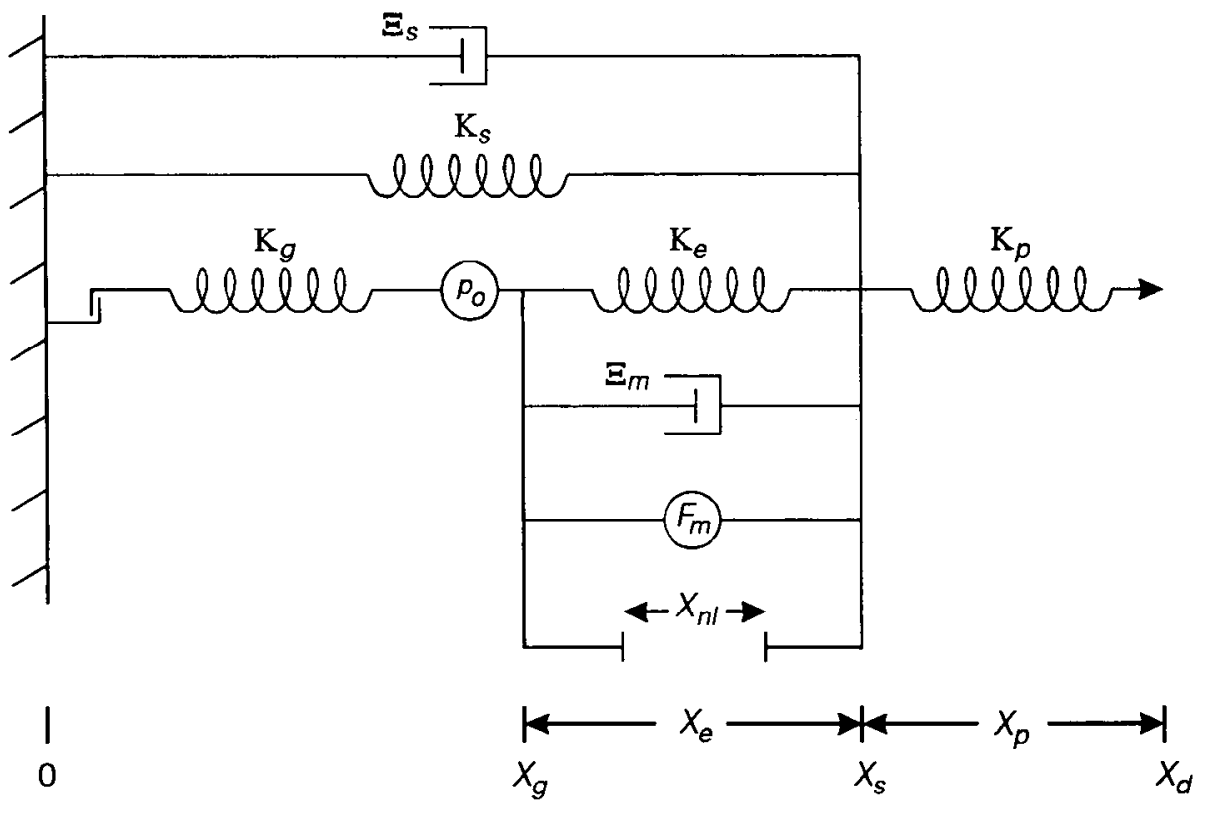

Figure 11. Mechanical model for adaptation, extended from Assad and Corey (1992) and Howard and Hudspeth (1987). The dashpot $\Xi_{s}$ represents viscous damping of the bundle. Intrinsic (pivoting) stiffness of the stereocilia is given stiffness $\mathbf{K}_{s}$, and the position of the stereociliary bundle is $X_{s}$. The stimulus probe has stiffness $K_{p}$, and the position of the distal end, the "drive," is $X_{d}$, so $X_{p}=X_{d}-X_{s}$. The gating spring has stiffness $\mathrm{K}_{g}$; its stretch from its zerotension length is $X_{g}$. In series with the gating spring is a "catch," to indicate that it does not work in compression, and a mechanically gated transduction channel, whose open probability $p_{0}$ responds to the tension $F_{g}=\mathrm{K}_{g} X_{g}$. The extent spring, with stiffness $\mathbf{K}_{e}$, connects to the gating spring at $X_{8}$, so its stretch is $X_{c}=X_{s}-X_{s}$. The motor is characterized by a dashpot, $\Xi_{m}$, in parallel with a force source, $F_{m}$; positive force is directed to the right, so it acts to stretch the gating spring and compress the extent spring. The negative limit of adaptation is represented as a "stop" of dimension $X_{n}$, such that $X_{e}$ $\geq X_{n i}$ at all times.
Some physical properties of $\mathrm{K}_{e}$ may be listed. As shown in the Appendix, its elastic stiffness constant (in $4 \mathrm{mM} \mathrm{Ca}^{2+}$ ) is about $120-160 \mu \mathrm{N} / \mathrm{m}$, if $K_{g}^{\prime}$ is $400-500 \mu \mathrm{N} / \mathrm{m}$ (Howard and Hudspeth, 1987, 1988; Jaramillo and Hudspeth, 1993). At steady state, the range of tensions it experiences is the same as that of the gating spring, since they are in series. The range of extensions it experiences is also similar to that of the gating spring, since a rapid positive deflection would stretch the gating spring, but then would stretch the extent spring by almost the same amount as the gating spring relaxed during adaptation. At steady state, the stretch of the extent spring will be fourfold that of the gating spring.

The negative limit represents a point beyond which the motor cannot climb. Although it could represent the point where the motor force balances the force in the extent spring, this is unlikely: when the gating spring is slack, this point would occur at a distance $X_{e}=F_{m} / \mathrm{K}_{e} \approx-1000 \mathrm{~nm}$. Since the resting compression in the extent spring (with $\mathrm{K}_{g}$ ) is $\sim 200 \mathrm{~nm}$, adaptation could proceed as far as $-800 \mathrm{~nm}$ before reaching this limit. Yet, $X_{n !}$ was generally between -100 and $-500 \mathrm{~nm}$, and was more negative than $-500 \mathrm{~nm}$ only if the bundle was given a large positive offset. In terms of movement along the side of a stereocilium in the tip-links model, $-500 \mathrm{~nm}$ corresponds to an upward distance of $60 \mathrm{~nm}$. This is the movement of the attachment point expected if the tip links are cut; a similar value was found in preliminary experiments (Shepherd et al., 1991). The negative limit behaves as a fixed barrier to the adaptation mechanism, and is unmoved by the forces accompanying large deflections, at least on the time scale studied in these experiments.

The extent spring and the negative limit presumably lie close to the structures mediating adaptation, perhaps as elements associated with the density marking the tip link's upper attachment. There are otherwise no obvious ultrastructural candidates for either component. The extent spring could consist of a meshwork of membrane and glycocalyx molecules, while the negative limit might be an actin-to-membrane linker. Alternatively, both components may correspond to specific molecular connections between the putative mechanoenzyme and the actin substrate; their dimensions are compatible with the possibility that they are individual molecules.

\section{Appendix}

Balance of forces

The forces acting on the position of the stereocilia, $X_{b}$, are

$$
\begin{aligned}
F_{d b} & =-\Xi_{b} d X_{s} / d t \\
F_{s} & =-\mathrm{K}_{s} X_{s} \\
F_{g} & =-\mathrm{K}_{g} X_{g}=-\mathrm{K}_{g}\left(X_{s}-X_{e}\right) \\
F_{p} & =\mathrm{K}_{p} X_{p}=\mathrm{K}_{p}\left(X_{d}-X_{s}\right) \\
F_{d b}+F_{s}+F_{g}+F_{p} & =0 .
\end{aligned}
$$

The viscous damping of the stereocilia, $\Xi_{b}$, is small in these experiments, allowing us to drop the term $F_{a b}$. The motor complex is in series with the gating spring, so tension in it is equal to gating spring tension and is represented by $F_{g}$. Then at any time

$$
X_{s}=\frac{\mathrm{K}_{g} X_{e}+\mathrm{K}_{p} X_{d}}{\mathrm{~K}_{s}+\mathrm{K}_{g}+\mathrm{K}_{p}} .
$$

The forces acting on the gating spring at position $X_{g}$ are

$$
\begin{aligned}
F_{d m} & =-\Xi_{m} d X_{e} / d t \\
F_{c} & =\mathrm{K}_{e} X_{c} \\
F_{g} & =-\mathrm{K}_{g} X_{g}=-\mathrm{K}_{g}\left(X_{s}-X_{e}\right) \\
F_{b}+F_{e}+F_{g}+F_{m} & =0 .
\end{aligned}
$$

Substituting for $X_{s}$ from Equation 2 above and solving for $X_{e}$ gives

$$
X_{e}(t)=\frac{X_{d} \mathrm{~K}_{g} \mathrm{~K}_{p}\left(1-e^{t / \tau}\right)-F_{m}\left(\mathrm{~K}_{s}+\mathrm{K}_{g}+\mathrm{K}_{p}\right)}{\left(\mathrm{K}_{s}+\mathrm{K}_{g}+\mathrm{K}_{p}\right)\left(\mathrm{K}_{g}+\mathrm{K}_{e}\right)-\mathrm{K}_{g}{ }^{2}},
$$


with a time constant $\tau=\Xi_{m} / \mathbf{K}_{s u b}$, where $\mathbf{K}_{s u b}=\mathbf{K}_{g}+\mathbf{K}_{e}-\mathbf{K}_{g}{ }^{2}$ / $\left(\mathrm{K}_{s}+\mathrm{K}_{g}+\mathrm{K}_{p}\right)$. The force generator $F_{m}$ contributes a constant offset in the extension of $X_{e}$.

\section{Stiff probe}

If the probe is very stiff, as was the case for the experiments in this report, then $\mathrm{K}_{p} \gg \mathrm{K}_{e}, \mathrm{~K}_{g}$, and $\mathrm{K}_{s}$. Consequently, $X_{s}=X_{d}$ at all times. Then

$$
X_{e}(t)-\frac{X_{s} \mathrm{~K}_{g}\left(1-e^{-t / r}\right)-F_{m}}{\mathrm{~K}_{g}+\mathrm{K}_{e}},
$$

and

$$
\tau=\frac{\Xi_{m}}{\mathbf{K}_{g}+\mathbf{K}_{e}} .
$$

At steady state, $X_{c \infty}=\left(\mathrm{K}_{g} X_{s}-F_{m}\right) /\left(\mathrm{K}_{g}+\mathrm{K}_{e}\right)$. It can be seen, then, that the extent of adaptation, expressed as a proportion of the bundle deflection, is $X_{e} / X_{s}=1 /(1+\phi)$, or 0.8 if $\phi=0.25$.

Adaptation may be similarly described as the stretch of the gating spring $X_{g}: X_{g}(t)=\left[X_{s}\left(\mathrm{~K}_{e}+\mathrm{K}_{g} e^{-t / \tau}\right)+F_{m}\right] /\left(\mathrm{K}_{g}+\mathrm{K}_{e}\right)$, with the same time constant as Equation 5. At steady state, $X_{g \infty}=$ $\left(X_{s} \mathrm{~K}_{e}+F_{m}\right) /\left(\mathrm{K}_{g}+\mathrm{K}_{e}\right)$. Note that the force on the transduction channels is $F_{g}=\mathrm{K}_{g} X_{g}$, and this is the essential stimulus to the cell.

If $X_{g}<0$ the tension in the gating spring goes to zero in this model, indicating that the gating springs do not act in compression. The movement of the motor's position is then $X_{e}(t)$ $=X_{e 0} e^{-t / \tau}$, where $\tau=\Xi_{m} / \mathrm{K}_{e}$, and $X_{e 0}$ is the initial value. This implies that the initial rate of adaptation for a negative deflection past the gating spring's slack point should grow with the "preloading" of the extent spring. The ensuing relaxation should follow an exponential time course governed by $\Xi_{m} / \mathrm{K}_{e}$, until $\mathrm{K}_{g}$ is engaged again; thereafter Equations 4 and 5 apply. If the gating springs did not reengage, or were removed from the system, the steady-state stretch of the extent spring would be $X_{e}=-F_{m} / \mathrm{K}_{e}$, or $X_{n l}$, whichever is limiting.

\section{Freestanding bundle}

If the bundle is freestanding $\left(\mathrm{K}_{p}=0\right)$, then for any perturbation that changes $X_{e}, X_{e}(t)=X_{e 0} e^{-t / \tau}-F_{m}\left(\mathrm{~K}_{s}+\mathrm{K}_{g}\right) / \mathrm{K}_{e q}$, where $X_{e 0}$ is the initial value, $\mathrm{K}_{e q}=\mathrm{K}_{s} \mathrm{~K}_{g}+\mathrm{K}_{g} \mathrm{~K}_{\rho}+\mathrm{K}_{e} \mathrm{~K}_{s}$, and the time constant is $\tau=\Xi_{m}\left(\mathrm{~K}_{s}+\mathrm{K}_{g}\right) / \mathrm{K}_{e q}$. At steady state, then, $X_{e \infty}=$ $-F_{m}\left(\mathrm{~K}_{s}+\mathrm{K}_{g}\right) / \mathrm{K}_{e q}$. Alternatively, this can be expressed in terms of the bundle position $X_{s}, X_{s}(t)=X_{s 0} e^{-t / \tau}-F_{m} \mathrm{~K}_{g} / \mathrm{K}_{e q}$, with the same time constant as for $X_{e}$. At steady state, $X_{s \infty}=-F_{m} \mathrm{~K}_{g}$ ' $\mathrm{K}_{e q}$. Lastly, the stretch of the gating spring for a freestanding bundle is $X_{g}=X_{s}-X_{e}$, so at steady state, $X_{g \infty}=F_{m} \mathrm{~K}_{s} / \mathrm{K}_{e q}$.

\section{Calculation of spring constants}

An interesting problem raised by this model is that the spring constants $\mathrm{K}_{g}$ and $\mathrm{K}_{s}$ are not the same as those estimated by Howard and Hudspeth, from a simpler model without $\mathrm{K}_{e}$. They applied the equivalent of a force $F$ to a hair bundle, and measured the initial $X_{i}$ and final $X_{f}$ bundle positions, before and after adaptation. In their model, $X_{l}=F /\left(\mathrm{K}_{g}{ }^{\prime}+\mathrm{K}_{s}{ }^{\prime}\right)$ and $X_{f}=$ $F / \mathrm{K}_{s}{ }^{\prime}$, where the primed spring constants indicate the Howard and Hudspeth values. In this model, $X_{i}=F /\left(\mathrm{K}_{g}+\mathrm{K}_{s}\right)$ but $X_{f}$ $=F\left(\mathrm{~K}_{g}+\mathrm{K}_{e}\right) / \mathrm{K}_{e q}$. These can be rearranged to give $\mathrm{K}_{g}=(1+$ $\phi) \mathrm{K}_{g}{ }^{\prime}$ and $\mathrm{K}_{s}=\mathrm{K}_{s}{ }^{\prime}-\phi \mathrm{K}_{g}{ }^{\prime}$, where $\phi=\mathrm{K}_{e} / \mathrm{K}_{g}$. The new $\mathrm{K}_{s}$ and $\mathrm{K}_{g}$ differ from the previous $\mathrm{K}_{s}^{\prime}$ and $\mathrm{K}_{g}$ ' by roughly $25 \%$ : taking $\mathrm{K}_{g}{ }^{\prime}$ as $430 \mu \mathrm{N} / \mathrm{m}$ and $\mathrm{K}_{s}{ }^{\prime}$ as $535 \mu \mathrm{N} / \mathrm{m}$ (Howard and Hudspeth,
1987, 1988; Jaramillo and Hudspeth, 1993) gives $\mathrm{K}_{g}$ as 538 $\mu \mathrm{N} / \mathrm{m}$ and $K_{s}$ as $428 \mu \mathrm{N} / \mathrm{m}$. This gives a value for $K_{e}$ of 134 $\mu \mathrm{N} / \mathrm{m}$, for $\phi=0.25$.

\section{Definitions of $\mathrm{F}_{\mathrm{m}}$ and $\mathrm{Z}_{\mathrm{m}}$}

How do the new motor parameters compare with expressions from the Assad and Corey (1992) model? With a stiff probe, the time constant of adaptation was $\tau=1 / S$. Under the same conditions, in this model, $\tau=\Xi_{m} /\left(\mathrm{K}_{g}+\mathrm{K}_{e}\right)$, so $B_{m}=\left(\mathrm{K}_{g}+\mathrm{K}_{e}\right) /$ $S$. Using the values for $\mathrm{K}_{g}$ and $\mathrm{K}_{e}$ from above, $\Xi_{m}$ has a value of $8.3 \mu \mathrm{N} \mathrm{sec} \mathrm{m}^{-1}$, at $-80 \mathrm{mV}$ in $4 \mathrm{mM} \mathrm{Ca}^{2+}$. Also, the resting stretch of the gating spring was $X_{g}=-X_{m}=-C / S$ in the previous model. Here the motor supplies a constant offset, $X_{e}=$ $-F_{m} /\left(\mathrm{K}_{g}+\mathrm{K}_{e}\right)$, so $F_{m}=\left(\mathrm{K}_{g}+\mathrm{K}_{e}\right) C / S=\Xi_{m} C$. Inserting the values obtained above, we get $F_{m}=130 \mathrm{pN}$, or about $22 \mathrm{pN}$ for each transduction and adaptation unit in a bundle of 60 stereocilia.

\section{References}

Assad JA, Corey DP (1992) An active motor model for adaptation in vertebrate hair cells. J Neurosci 12:3291-3309.

Assad JA, Ilacohen N, Corey DP (1989) Voltage dependence of adaptation and active bundle movement in bullfrog saccular hair cells. Proc Natl Acad Sci USA 86:2918-2922.

Assad JA, Shepherd GMG, Corey DP (1991) Tip-link integrity and mechanical transduction in vertebrate hair cells. Neuron 7:985-994.

Baird RA (1992) Morphological and electrophysiological properties of hair cells in the bullfrog utriculus. Ann NY Acad Sci 656:12-26.

Baird RA, Lewis ER (1986) Correspondences between afferent innervation patterns and response dynamics in the bullfrog utricle and lagena. Brain Res 369:48-64.

Corey DP, Hudspeth AJ (1980) Mechanical stimulation and micromanipulation with piezoelectric bimorph elements. J Neurosci Methods 3:183-202.

Corey DP, Hudspeth AJ (1983a) Analysis of the microphonic potential of the bullfrog's sacculus. J Neurosci 3:942-961.

Corey DP, Hudspeth AJ (1983b) Kinetics of the receptor current in bullfrog saccular hair cells. J Neurosci 3:962-976.

Corey DP, Hacohen N, Huang PL, Assad JA (1989) Hair cell stereocilia bend at their bases and touch at their tips. Soc Neurosci Abstr 15:208a.

Crawford AC, Evans MG, Fettiplace R (1989) Activation and adaptation of transducer currents in turtle hair cells. J Physiol (Lond) 419: 405-434.

Eatock RA, Corey DP, Hudspeth AJ (1987) Adaptation of mechanoelectrical transduction in hair cells of the bullfrog's sacculus. J Neurosci 7:2821-2836.

Furukawa T, Matsuura S (1978) Adaptive rundown of excitatory postsynaptic potentials at synapses between hair cells and eighth nerve fibres in the goldfish. J Physiol (Lond) 276:193-209.

Gillespie PG, Hudspeth AJ (1993) Adenine nucleoside diphosphates block adaptation of mechanoelectrical transduction in hair cells. Proc Natl Acad Sci USA 90:2710-2714.

Goldberg JM, Fernández C (1984) The vestibular system. In: Handbook of physiology, Vol 3 (Brookhart JM, Mountcastle VB, eds), pp 977-1021. Bethesda, MD: American Physiological Society.

Hacohen N, Assad JA, Smith WJ, Corey DP (1989) Regulation of tension on hair-cell transduction channels: displacement and calcium dependence. J Neurosci 9:3988-3997.

Hill AV (1938) The heat of shortening and the dynamic constants of muscle. Proc R Soc Lond [Biol] 126:136-195.

Holton T, Hudspeth AJ (1986) The transduction channel of hair cells from the bull-frog characterized by noise analysis. J Physiol (Lond) 375:195-227.

Howard J, Hudspeth AJ (1987) Mechanical relaxation of the hair bundle mediates adaptation in mechanoelectrical transduction by the bullfrog's saccular hair cell. Proc Natl Acad Sci USA 84:3064-3068.

Howard J, Hudspeth AJ (1988) Compliance of the hair bundle associated with gating of mechanoelectrical transduction channels in the bullfrog's saccular hair cell. Neuron 1:189-199.

Hudspeth AJ (1989) How the ear's works work. Nature 341:397-404. 
Hudspeth AJ, Jacobs R (1979) Stereocilia mediate transduction in vertebrate hair cells. Proc Natl Acad Sci USA 76:1506-1509.

Huxley AF, Simmons RM (1971) Proposed mechanism of force generation in striated muscle. Nature 233:533-538.

Jacobs RA, Hudspeth AJ (1990) Ultrastructural correlates of mechanoelectrical transduction in hair cells of the bullfrog's internal ear. Cold Spring Harbor Symp Quant Biol 55:547-561.

Jaramillo F, Hudspeth AJ (1993) Displacement-clamp measurement of the forces exerted by gating springs in the hair bundle. Proc Natl Acad Sci USA 90:1330-1334.

Kimitsuka T, Ohmori $H$ (1992) The effect of caged calcium release on the adaptation of the transduction current in chick hair cells. J Physiol (Lond) 458:27-40.

Kros CJ, Rüsch A, Richardson GP (1992) Mechano-electrical transducer currents in hair cells of the cultured neonatal mouse cochlea. Proc R Soc Lond [Biol] 249:185-193.
Lewis ER, Baird RA, Leverenz EL, Koyama H (1982) Inner ear: dye injection reveals peripheral origins of specific sensitivities. Science 215:1641-1643.

Liberman MC (1987) Chronic ultrastructural changes in acoustic trauma: serial-section reconstruction of stereocilia and cuticular plates. Hearing Res 26:65-88.

Pickles JO, Corey DP (1992) Mechano-electrical transduction by hair cells. Trends Neurosci 15:254-259.

Pickles JO, Comis SD, Osborne MP (1984) Cross-links between stereocilia in the guinea pig organ of Corti, and their possible relationship to sensory transduction. Hearing Res 15:103-112.

Shepherd GMG, Assad JA, Parakkal M, Kachar B, Corey DP (1991) Movement of the tip-link attachment is correlated with adaptation in bullfrog saccular hair cells. J Gen Physiol 98:25a. 